\title{
Decomposição de resíduo carbonífero e de curtume in vitro em argissolo vermelho distrófico típico
}

\author{
Carboniferous residue decomposition and in vitro tannery in typical red dystrophic \\ argissolo
}

\author{
Cláudio Kray ${ }^{1}$, Robson Andreazza ${ }^{2}$, Marino Tedesco ${ }^{3}$, Carlos Bissani ${ }^{4}$, Leandro Bortolon ${ }^{5}$, \\ Elisandra Bortolon ${ }^{6}$, Clésio Gianello ${ }^{7}$ \\ 1, 2,3,4,5,6,7 Universidade Federal de Pelotas, UFPel, Pelotas, RS - Brasil
}

\begin{abstract}
Resumo
Os resíduos gerados na exploração de carvão mineral e no curtume de peles são produtos potencialmente poluentes em várias regiões do Brasil, principalmente nos estados do Rio Grande do Sul e de Santa Catarina. O objetivo do trabalho foi avaliar a taxa de degradação e a variação de nitrogênio mineral e $\mathrm{pH}$ do solo pela aplicação de resíduos de curtume e carbonífero no solo. O experimento foi conduzido em frascos respirométricos em um Argissolo Vermelho Distrófico típico que estava sob campo nativo. Foram aplicados oito tratamentos sendo testados o lodo de efluentes (LC) e a serragem cromada (SC) de curtume e resíduo carbonífero (RC). A adição de lodo de curtume (LC + PK) promoveu uma degradação de $23,3 \%$ do carbono adicionado após 88 dias de incubação. O lodo de curtume foi eficiente para aumentar o $\mathrm{pH}$ do solo a $\mathrm{pH}$ 6. O lodo de curtume pode ser utilizado para a neutralização da acidez do solo gerada pelo descarte de resíduo carbonífero contendo alto teor de enxofre reduzido. A serragem cromada pode ser descartada no solo, desde que haja uma calagem e adubação com nitrogênio, fósforo e potássio em quantidades adequadas para o desenvolvimento das culturas. O resíduo carbonífero apresenta grande potencial de acidificação do solo e baixa taxa de decomposição.
\end{abstract}

Palavras-chave: cromo, lodo de curtume, degradação de resíduos, serragem cromada, atividade microbiana.

\begin{abstract}
The waste generated in coal mining and tannery are potential pollutants in various regions of Brazil, especially in the states of Rio Grande do Sul and Santa Catarina. The aim of this study was to evaluate the degradation rate and the variation of mineral nitrogen and soil pH after applying tannery waste and coal mining in the soil. The experiment was conducted in respirometric flasks in a Ultisol that was under native pasture. Eight treatments were applied and tested the tannery sludge (LC) and leather shavings (SC) tannery waste coal (RC). The addition of tannery sludge $(L C+P K)$ promoted degradation of $23.3 \%$ of the carbon added after 88 days of incubation. Tannery sludge was effective to increase the soil $p H$ to $\mathrm{pH}$ 6. Tannery sludge can be used to neutralize soil acidity generated by the disposal of coal mining waste containing high reduced sulfur. The leather shavings can be disposed on the soil since it there is fertilization with nitrogen, phosphorus and potassium in adequate amounts for crop development. The coal mining waste has great potential for soil acidification and low decomposition rate.
\end{abstract}

Key-words: chromium, tannery sludge, residues degradation, coal waste, microbial activity. 


\section{Introdução}

A indústria coureiro-calçadista e a mineração do carvão são atividades importantes na economia do Estado do Rio Grande do Sul. Entretanto, essas atividades geram quantidades consideráveis de resíduos. A aplicação de resíduos de atividades industriais em solo agrícola vem sendo utilizada de forma crescente em nível mundial, sendo uma alternativa ambiental e econômica viável (KRAY et al., 2011; SEGATTO et al., 2012). Devido suas características químicas e biológicas, o solo pode proporcionar as condições necessárias para a biodegradação de resíduos orgânicos e a retenção dos metais pesados e outros poluentes (MOREIRA e SIQUEIRA, 2006).

O material orgânico pode disponibilizar nutrientes, como o nitrogênio, o fósforo e o potássio para as plantas e os microrganismos, e melhorar atributos químicos e físicos do solo pela adição de matéria orgânica (KRAY et al., 2011; KROB et al., 2011; LAUSCHNER et al., 2012; SEGATTO et al., 2012). Entretanto, é necessário o monitoramento de possíveis efeitos negativos decorrentes da aplicação destes resíduos ao solo, tais como, a contaminação por metais pesados, a redução na atividade microbiana e a contaminação das águas subterrâneas por nitrato $\left(\mathrm{NO}_{3}\right)$.

A degradação de resíduos pela respirometria avaliando a atividade microbiana é uma prática muito utilizada e com grande utilidade na avaliação ambiental na utilização de resíduos (STOTZKY, 1965; MOREIRA e SIQUEIRA, 2006). Além disso, pode ser uma alternativa para avaliar a degradação de compostos orgânicos como resíduos carboníferos e de lodo de curtume (KRAY et al., 2011; LAUSCHNER et al., 2012; SEGATTO et al., 2012) e também outros resíduos como diesel e biodiesel (MEYER et al., 2012; COLLA et al., 2013).

O lodo de curtume pode ser utilizado para a correção do $\mathrm{pH}$ de solos ácidos e como fonte de nitrogênio para as plantas (BIANCHIN, 2011). Vários estudos realizados em condições de casa de vegetação e a condições de campo demonstraram a viabilidade do uso do lodo para as culturas comerciais, por exemplo, milho e soja (STOMBERG et al., 1984; FERREIRA et al., 2003), espécies florestais como acácia negra, eucalipto (TEIXEIRA, 1981; FISCH, 1992; FISCH, 1994) e hortaliças (FIGLIOLIA et al., 1992; CAVALLET et al., 2007).

Os processos de extração, beneficiamento e utilização do carvão mineral têm elevado potencial poluidor, pelo grande volume de resíduos gerados. $\mathrm{O}$ potencial poluidor do rejeito carbonífero está associado à oxidação da pirita. Na recuperação das áreas mineradas, o estabelecimento da vegetação é dificultado pela acidez do solo e baixo nível de fertilidade do sis- tema. Vários estudos de longa duração têm avaliado o potencial de lodos de esgoto como corretivo da acidez de resíduos carboníferos (BIANCHIN, 2011). Além disso, o mesmo autor relata que o efeito corretivo do lodo de curtume sobre o $\mathrm{pH}$ e outros atributos ainda pode ser observado no solo na camada superficial, com expansão também para a camada subsuperficial, no tratamento com maior dose aplicada.

Outro estudo com experimento a campo com aplicação de 106 ton ha $^{-1}$ de resíduo carbonífero no solo, e a mesma dose mais 21,25 ton ha $^{-1}$ de lodo de ETE de curtume, obteve valores de $\mathrm{pH}$ do solo e rendimentos de grãos de milho e soja maiores no tratamento com lodo, sendo esses valores semelhantes aos determinados no tratamento com calcário e adubação mineral, sem aplicação de resíduo carbonífero (FERREIRA et al., 2003). Portanto, o descarte de resíduos no solo deve ser feito considerando a taxa de degradação e a composição química. Desta forma, têm grande importância os estudos da taxa de decomposição desses resíduos no solo. Assim, este trabalho foi desenvolvido para avaliar a taxa de degradação e a variação de nitrogênio mineral e pH do solo pela aplicação de resíduos de curtume e carbonífero no solo.

\section{Material e métodos}

O estudo foi realizado em condições controladas no Laboratório de Análises de Solos da UFRGS. O experimento foi conduzido em frascos respirométricos em um Argissolo Vermelho distrófico típico (EMBRAPA, 2006), que estava sob campo nativo (na estação experimental da UFRGS, em Junho de 2006), devido suas características nativas, não apresentando nenhuma forma de contaminação. Os atributos físicoquímicos do solo (Tabela 1) foram determinados conforme metodologia descrita em Tedesco et al. (1995) utilizando-se amostras secas $\left(65^{\circ} \mathrm{C}\right)$ trituradas em cadinho de porcelana. O resíduo carbonífero foi obtido da COPELMI (empresa mineradora de carvão), localizada no município de Butiá (RS). O lodo de efluentes e a serragem cromada de curtume foram obtidos na Unidade de Tratamento de Resíduos (UTRESA), de Estância Velha (RS). As características químicas dos resíduos acima descritos são mostradas na Tabela 2. As determinações foram efetuadas conforme a metodologia descrita por Tedesco et al. (1995).

Para avaliar a taxa de decomposição dos resíduos no solo foram usadas $200 \mathrm{~g}$ de solo (base seca) para cada unidade experimental. Os tratamentos utilizados foram (Tabela 3): 1- Testemunha; 2- NPK mais calcário para atingir pH 6,0 (NPK + calcário); 3- (LC) em quantidade adequada para atingir $\mathrm{pH}$ 6,0 
mais PK (LC + PK); 4- Duas vezes a quantidade de lodo de curtume utilizada no tratamento 3 mais PK (2 LC + PK); 5- Resíduo carbonífero (RC) na quantidade de 56 ton ha ${ }^{-1}$ mais NPK (RC + NPK); 6- Resíduo carbonífero (RC) na quantidade de 56 ton ha ${ }^{-1}$ mais lodo de curtume na quantidade utilizada no tratamento 3 mais PK (RC + LC + PK); 7a- Serragem cromada (SC) na quantidade de 30 ton ha ${ }^{-1}$ mais NPK e calcário (SC + NPK + calcário); 7b- Serragem cromada (SC) na quantidade de 30 ton ha ${ }^{-1}$ mais PK e calcário (SC + PK + calcário) e 8- Cromo mineral (500 kg de $\mathrm{Cr}^{3+}$ $\mathrm{ha}^{-1}$ ) mais lodo de curtume na quantidade utilizada no tratamento 3, mais PK $\left(\mathrm{Cr}_{\min }+\mathrm{LC}+\mathrm{PK}\right)$.

A adubação mineral no solo das unidades experimentais, foi feita pela aplicação de 160, 120 e $50 \mathrm{~kg} \mathrm{ha}^{-1}$ de $\mathrm{N}$ (na forma de uréia), $\mathrm{P}_{2} \mathrm{O}_{5}$ (na forma de superfosfato triplo) e $\mathrm{K}_{2} \mathrm{O}$ (na forma de $\mathrm{KCl}$ ), respectivamente, conforme a recomendação da SBCS/ NRS (1995). A calagem foi feita em dose adequada para atingir $\mathrm{pH}$ 6,0 (mistura de $\mathrm{CaCO}_{3}+\mathrm{MgCO}_{3}$ ( 3:1 )) com base nos teores de alumínio trocável e de matéria orgânica do solo, na quantidade de 6,3 ton ha ${ }^{-1}$ de calcário (PRNT 100\%) de acordo com a recomendação (SBCS/NRS, 1995).

Tabela 3. Tratamentos utilizados no experimento com Argissolo.

\begin{tabular}{|c|c|c|c|c|}
\hline Tratamento & Resíduo & Cromo Min & Calcário** & Adubação \\
\hline 1) Testemunha & $\mathrm{na}^{*}$ & na & na & na \\
\hline 2) NPK + calcário & na & na & $\mathrm{pH} 6,0$ & NPK \\
\hline 3) $\mathrm{LC}$ & Lodo de curtume para corrigir solo à $\mathrm{pH} 6,0$ & na & na & na \\
\hline 4) $-2 \mathrm{LC}+\mathrm{PK}$ & $\begin{array}{c}2 \times \text { Lodo de curtume adicionado no } \\
\text { Trat. } 3\end{array}$ & na & na & PK \\
\hline 5) $\mathrm{RC}+\mathrm{NPK}$ & Resíduo carbonífero (56 ton. ha-1) & na & na & NPK \\
\hline 6) $\mathrm{RC}$ & Resíduo carbonífero & na & na & na \\
\hline 7a) SC + NPK + calcário & Serragem cromada (30 ton ha-1) & na & $\mathrm{pH} 6,0$ & NPK \\
\hline 7b) SC + PK + calcário & Serragem cromada (SC) 30 ton $\mathrm{ha}^{-1}$ & na & $\mathrm{pH} 6,0$ & PK \\
\hline 8) $\mathrm{Cr}_{\min }+\mathrm{LC}+\mathrm{PK}$ & Lodo de curtume $=$ utilizado no Trat. 3 & $\begin{array}{c}500 \mathrm{~kg} \text { de } \mathrm{Cr}^{3+} \\
\mathrm{ha}^{-1}\end{array}$ & na & PK \\
\hline
\end{tabular}

*na significa não adicionado.

** calcário ou resíduo calculado para neutralizar o solo a pH 6. 
O lodo de curtume foi adicionado em quantidade equivalente à dose de calcário para atingir $\mathrm{pH}$ 6,0 . O lodo de curtume (com 31,5\% de valor de neutralização, expresso em peso seco a $65^{\circ} \mathrm{C}$ ) foi aplicado na quantidade de 22,4 ton ha- ${ }^{-1}$ (base seca), equivalente a 6,3 t ha ${ }^{-1}$ de calcário (PRNT 100\%). O resíduo carbonífero foi aplicado na quantidade de 56 ton ha- ${ }^{-1}$, visando à neutralização da alcalinidade do solo.

Com relação ao cromo, na forma mineral $\left(\mathrm{Cr}_{\text {min }}\right)$ foi adicionado o sulfato de cromo (Cr III) na quantidade de $500 \mathrm{~kg} \mathrm{ha}^{-1} \mathrm{de} \mathrm{Cr}$. A serragem cromada foi adicionada na quantidade de 30 ton ha-1 (com 2,2\% de $\mathrm{Cr}$ - em peso seco a 75\%) supriu $660 \mathrm{~kg} \mathrm{de} \mathrm{Cr} \mathrm{ha}^{-1}$.

Após a homogeneização, o solo com os tratamentos aplicados foi acondicionado em frascos respirométricos de vidro (1L) adicionando-se a seguir água destilada até atingir $80 \%$ da capacidade máxima de retenção de água do solo, que foi mantida a mesma umidade até o fim do experimento, pela avaliação da variação da massa dos frascos. Na superfície do solo de cada frasco de incubação, foi colocado um suporte de ferro suspenso um copo plástico $(50 \mathrm{~mL})$ contendo $20 \mathrm{~mL}$ de $\mathrm{NaOH} 0,5 \mathrm{~mol} \mathrm{~L}^{-1}$. Os frascos respirométricos foram mantidos hermeticamente fechados e abertos somente para as avaliações. Além disso, foram mantidos três frascos respirométricos sem solo, representando a prova em branco.

A determinação das quantidades de $\mathrm{CO}_{2}$ liberado pela atividade microbiana, em função dos tratamentos, foi feita aos 2, 4, 7, 13, 20, 26, 32, 39, 47, $53,60,67,74,81$ e aos 88 dias de incubação. A cada avaliação, os frascos respirométricos foram abertos e na solução de $\mathrm{NaOH}$ foi adicionado $1,0 \mathrm{~mL}$ de $\mathrm{BaCl}_{2}$ (25\%) e 3 gotas de indicador fenolftaleína. Posteriormente, foi feita a titulação do excesso de $\mathrm{NaOH}$ com solução de $\mathrm{HCl}$ 0,5 mol L-1 (STOTZKY, 1965). Ao final das titulações em cada período de avaliação, um novo copo plástico contendo $\mathrm{NaOH} 0,5 \mathrm{~mol} \mathrm{~L}^{-1}$ foi colocado novamente dentro dos frascos respirométricos.

A liberação de $\mathrm{CO}_{2}$ (mg de C- $\mathrm{CO}_{2} \mathrm{~kg}^{-1}$ de solo) pela atividade microbiana do solo pela aplicação dos resíduos, foi calculada pela fórmula:

$$
\text { mg C }-\mathrm{CO}_{2}=(\mathrm{B}-\mathrm{V}) \times \mathrm{M} \times \mathrm{E} \text {. }
$$

Os termos da equação são: B é o volume de $\mathrm{HCl}$ necessário para titular o excedente de $\mathrm{NaOH}$ da prova em branco; $\mathrm{V}$ é o volume de $\mathrm{HCl}$ necessário para titular o excedente de $\mathrm{NaOH}$ da amostra; $\mathrm{M}$ é a molaridade do $\mathrm{HCl}\left(0,5 \mathrm{~mol} \mathrm{~L}^{-1}\right)$; E é o peso equivalente grama do carbono (6).

A quantidade de $\mathrm{CO}_{2}$ liberado pela incorporação do substrato orgânico foi obtida pela diferença entre o $\mathrm{CO}_{2}$ determinado no solo em que foi adicionado resíduo, menos o determinado em um tratamento com solo sem adição de resíduo (testemunha).

Na última data de avaliação de $\mathrm{CO}_{2}$ (88 dias), foi retirada uma amostra de solo de cada unidade experimental e determinado $\mathrm{pH}$ do solo e os teores de nitrogênio inorgânico $\left(\mathrm{NH}_{4}^{+}\right.$e $\left.\mathrm{NO}_{3}^{-}\right)$, conforme metodologia descrita em Tedesco et al. (1995).

A análise dos dados foi feita pela análise da variância individual e conjunta dos dados, o teste de significância foi feito pelo teste F. Quando significativo, foi feito a comparação múltipla de médias pelo teste de Tukey, ao nível de $5 \%$ de probabilidade.

Tabela 1. Caracterização física e química do Argissolo Vermelho arênico distrófico do local do experimento $(0-20 \mathrm{~cm})$.

\begin{tabular}{|c|c|}
\hline Atributos $^{1}$ & Concentrações \\
\hline Argila $\left(\mathrm{g} \mathrm{kg}^{-1}\right)$ & 250 \\
\hline Matéria orgânica $\left(\mathrm{g} \mathrm{kg}^{-1}\right)$ & 25,0 \\
\hline Nitrogênio total $\left(\mathrm{g} \mathrm{kg}^{-1}\right)$ & 1,12 \\
\hline $\mathrm{C} / \mathrm{N}$ total & 13,1 \\
\hline pH em água & 4,9 \\
\hline Índice SMP & 5,9 \\
\hline P disponível $\left(\mathrm{mg} \mathrm{dm}^{-3}\right)$ & 3 \\
\hline $\mathrm{K}$ disponível $\left(\mathrm{mg} \mathrm{dm}^{-3}\right)$ & 153 \\
\hline $\mathrm{Al}$ trocável $\left(\mathrm{mmol}_{\mathrm{c}} \mathrm{dm}^{-3}\right)$ & 6,0 \\
\hline Ca trocável $\left(\mathrm{mmol}_{\mathrm{c}} \mathrm{dm}^{-3}\right)$ & 16,8 \\
\hline Mg trocável $\left(\mathrm{mmol}_{\mathrm{c}} \mathrm{dm}^{-3}\right)$ & 12,5 \\
\hline CTC $\left(\mathrm{mmol}_{\mathrm{c}} \mathrm{dm}^{-3}\right)$ & 53 \\
\hline $\mathrm{H}+\mathrm{AL}\left(\mathrm{mmol}_{\mathrm{c}} \mathrm{dm}^{-3}\right)$ & 18,5 \\
\hline \multicolumn{2}{|l|}{ \% de saturação da CTC: } \\
\hline por Bases & 63 \\
\hline por $\mathrm{Al}$ & 11 \\
\hline S extraível $\left(\mathrm{mg} \mathrm{dm}^{-3}\right)$ & 8,9 \\
\hline B extraível $\left(\mathrm{mg} \mathrm{dm}^{-3}\right)$ & 0,5 \\
\hline Mn trocável $\left(\mathrm{mg} \mathrm{dm}^{-3}\right)$ & 26 \\
\hline
\end{tabular}

${ }^{1}$ Determinações efetuadas conforme metodologia descrita por Tedesco et al., (1995). 
Tabela 2. Características físico-químicas dos resíduos utilizados na aplicação e na reaplicação dos tratamentos.

\begin{tabular}{|c|c|c|c|}
\hline Características* & Lodo de curtume & Serragem cromada & Resíduo carbonífero \\
\hline Teor de sólidos $\left(\mathrm{g} \mathrm{kg}^{-1}\right)$ & 300 & 480 & 925 \\
\hline pH em água & 7,7 & 3,3 & 7,0 \\
\hline Carbono orgânico $\left(\mathrm{g} \mathrm{kg}^{-1}\right)$ & 203,8 & 326,0 & 183,8 \\
\hline Nitrogênio total $\left(\mathrm{g} \mathrm{kg}^{-1}\right)$ & 32,5 & 119,7 & 2,3 \\
\hline Fósforo total ( $\left.\mathrm{g} \mathrm{kg}^{-1}\right)$ & 2,5 & 0,4 & 0,3 \\
\hline Potássio total $\left(\mathrm{g} \mathrm{kg}^{-1}\right)$ & 0,12 & 0,12 & 0,06 \\
\hline Cálcio total $\left(\mathrm{g} \mathrm{kg}^{-1}\right)$ & 22,0 & 18,0 & 19,0 \\
\hline Magnésio total $\left(\mathrm{g} \mathrm{kg}^{-1}\right)$ & 3,50 & 0,19 & 0,68 \\
\hline Enxofre total $\left(\mathrm{g} \mathrm{kg}^{-1}\right)$ & 15,0 & 15,9 & 85,0 \\
\hline Cobre total $\left(\mathrm{mg} \mathrm{kg}^{-1}\right)$ & 34 & 3 & 25 \\
\hline Zinco total $\left(\mathrm{mg} \mathrm{kg}^{-1}\right)$ & 176 & 2 & 126 \\
\hline Manganês total (mg kg-1) & 128 & $<3$ & 194 \\
\hline Sódio total $\left(\mathrm{g} \mathrm{kg}^{-1}\right)$ & 8,1 & 4,3 & ND \\
\hline Cromo total $\left(\mathrm{g} \mathrm{kg}^{-1}\right)$ & 22,2 & 24,0 & 0,3 \\
\hline Cádmio total $\left(\mathrm{mg} \mathrm{kg}^{-1}\right)$ & 0,14 & $<0,01$ & 12,85 \\
\hline Níquel total $\left(\mathrm{mg} \mathrm{kg}^{-1}\right)$ & 14,50 & 3,15 & 19,20 \\
\hline Poder de neutralização (\%) & 31,5 & ND & ND \\
\hline
\end{tabular}

*Determinações feitas no material seco a $75^{\circ} \mathrm{C}$, à exceção do $\mathrm{pH}$.

$\mathrm{ND}=$ não determinado.

\section{Resultados e discussão}

A adição de resíduos de curtume e carbonífero no solo alterou a liberação de $\mathrm{CO}_{2}$ em relação ao tratamento testemunha (somente solo) (Figura 1). As quantidades cumulativas de $\mathrm{C}-\mathrm{CO}_{2}$ liberadas aos 13, 32, 60 e 88 dias de incubação são apresentadas na Tabela 3. A menor quantidade de $\mathrm{C}-\mathrm{CO}_{2}$ evoluída foi determinada na testemunha, devido neste tratamento somente ocorrer à mineralização da matéria orgânica do solo (mineralização basal). A mineralização foi potencializada no tratamento com adubação mineral, pelo aumento do $\mathrm{pH}$ (Tabela 4) e suprimento de nutrientes.

A atividade microbiana do solo é um indicar importante na degradação dos resíduos, pois a microbiota do solo promove degradação, e consequentemente, acelera a mineralização dos nutrientes (ATLAS e BARTHA, 1997; MOREIRA e SIQUEIRA, 2006). Contudo, um aspecto deve ser levado em conta, quando há um elemento tóxico, como o cromo, por exemplo, a microbiota normal do solo decresce sua atividade e a microbiota resistente aumenta, podendo esta, ser ou não mais eficiente na degradação. No caso deste trabalho, não ocorreu decréscimo na atividade microbiana quando houve a adição do dobro da dose de lodo de curtume, ocorrendo sim, um aumento significativo na degradação do mesmo (Figura 1). Isto demonstra que mesmo em altas doses de lodo de curtume contendo cromo, não afetou negativamente a atividade microbiológica deste solo. 


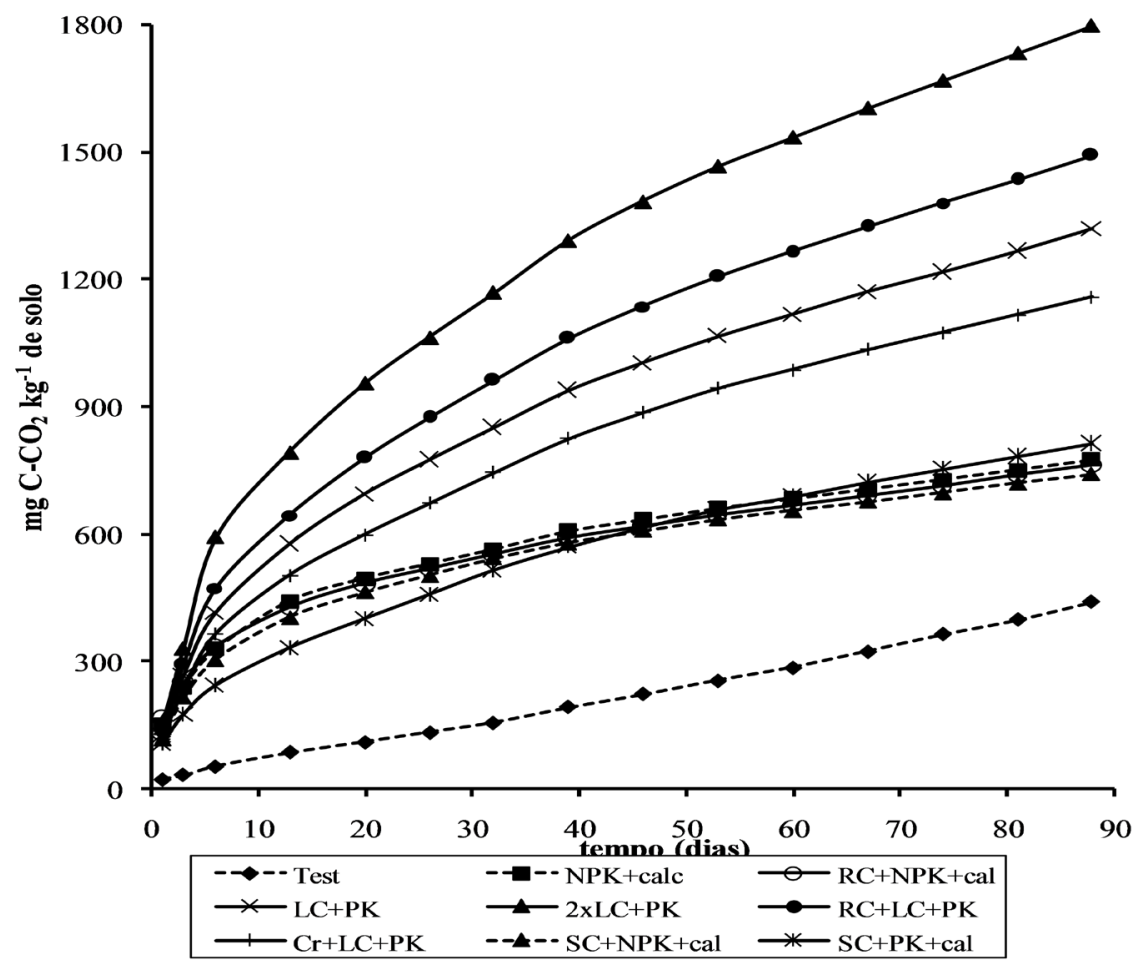

Figura 1. Liberação acumulada de carbono (C-CO2) no solo após 88 dias de incubação com os diferentes tratamentos.

Após o período inicial (32 dias), a liberação de $\mathrm{CO}_{2}$ demonstrou uma estabilização na evolução de $\mathrm{CO}_{2} \mathrm{em}$ alguns tratamentos como nos tratamentos com $2 \mathrm{xLC}+\mathrm{PK}, \mathrm{RC}+\mathrm{LC}+\mathrm{PK}, \mathrm{Cr}+\mathrm{LC}+\mathrm{PK}, \mathrm{e} \mathrm{LC}+\mathrm{PK}$ (Figura 1). Os valores determinados nos tratamentos com adubação mineral ou adição de resíduos orgânicos foram sempre superiores ao tratamento testemunha, pois a adição de adubação mineral e resíduos utilizados favorecem a atividade microbiana. Isto é devido ao "efeito priming", onde há uma atividade mais intensa quando grandes quantidades de material orgânico são adicionadas, e a fração mais lábil e de fácil decomposição é prontamente decomposta em uma taxa muito maior. Assim, após esta fase, há uma estabilização da decomposição. Efeitos semelhantes são descritos por vários autores e explicados assim na literatura (ATLAS e BARTHA, 1997; MOREIRA e SIQUEIRA, 2006).

A decomposição do lodo de curtume no solo pode ser avaliada pelo acréscimo de liberação de $\mathrm{C}-\mathrm{CO}_{2}$, que aumentou com a quantidade adicionada (LC + PK e 2 LC + PK) e com relação a Testemunha. No tratamento com a adição de 22,4 ton ha-1 de lodo de curtume ( $\mathrm{LC}+\mathrm{PK}$ ) foi determinada uma emissão total de $1.292 \mathrm{mg}$ de $\mathrm{C}-\mathrm{CO}_{2} \mathrm{~kg}^{-1} \mathrm{o}$ que representou $23,3 \%$ do carbono adicionado com o tratamento no período de 88 dias de incubação (Tabela 5).
Tabela 3. Liberação acumulada de carbono (mg de C-CO2 kg-1 de solo) em vários períodos da incubação (média de três repetições).

\begin{tabular}{|c|c|c|c|c|}
\hline Tratamento & 13 dias & 32 dias & 60 dias & 88 dias \\
\hline $\begin{array}{c}1 \text { - } \\
\text { Testemunha }\end{array}$ & 81 hD* & 151 hC & $281 \mathrm{hB}$ & $435 \mathrm{hA}$ \\
\hline $\begin{array}{l}2 \text { - NPK + } \\
\text { calcário }\end{array}$ & $425 \mathrm{eD}$ & $548 \mathrm{eC}$ & 667 efB & $759 \mathrm{fA}$ \\
\hline $3-\mathrm{LC}+\mathrm{PK}$ & $551 \mathrm{cD}$ & 826 cC & $1091 \mathrm{cB}$ & $1292 \mathrm{cA}$ \\
\hline $\begin{array}{c}4-2 \times \mathrm{LC}+ \\
\text { PK }\end{array}$ & $707 \mathrm{aD}$ & $1083 \mathrm{aC}$ & $1449 \mathrm{aB}$ & $1713 \mathrm{aA}$ \\
\hline $\begin{array}{c}5 \text { - RC + NPK } \\
\text { + calcário }\end{array}$ & $414 \mathrm{eD}$ & 538 efC & $652 \mathrm{fgB}$ & $748 \mathrm{fA}$ \\
\hline $\begin{array}{c}6-\mathrm{RC}+\mathrm{LC} \\
+\mathrm{PK}\end{array}$ & $613 \mathrm{bD}$ & 932 bC & $1234 \mathrm{bB}$ & $1462 \mathrm{bA}$ \\
\hline $\begin{array}{c}\text { 7a - SC } \\
+\mathrm{NPK}+ \\
\text { calcário }\end{array}$ & $388 \mathrm{fD}$ & $527 \mathrm{fC}$ & $639 \mathrm{gB}$ & $725 \mathrm{gA}$ \\
\hline $\begin{array}{c}7 \mathrm{~b}-\mathrm{SC}+\mathrm{PK} \\
\text { + calcário }\end{array}$ & $319 \mathrm{gD}$ & $504 \mathrm{gC}$ & 676 eB & $801 \mathrm{eA}$ \\
\hline $\begin{array}{c}8-\mathrm{Cr}+\mathrm{LC} \\
+\mathrm{PK}\end{array}$ & $480 \mathrm{dD}$ & $722 \mathrm{dC}$ & $965 \mathrm{~dB}$ & $1137 \mathrm{dA}$ \\
\hline
\end{tabular}

*Médias seguidas de mesma letra minúscula e maiúscula não diferem entre tratamentos e épocas, respectivamente, pelo teste de Tukey $(\mathrm{p}<0,05)$. 
A liberação de $\mathrm{C}-\mathrm{CO}_{2}$ no tratamento com adição de serragem cromada (SC + PK + Calcário) foi inferior à determinada no tratamento com adubação mineral (NPK + Calcário) até o período de 55 dias do inicio da incubação. Ao final de 88 dias determinouse uma decomposição de somente $0,83 \%$ do carbono adicionado. Castilhos et al. (2002) determinou, entretanto, uma liberação de $15 \%$ do carbono adicionado pela serragem cromada num período de 366 dias, com adição de nitrogênio (média de três solos). A dificuldade de decomposição da serragem cromada pode ser justificada pelo processo de curtimento do couro que torna este material pouco suscetível ao ataque microbiano. A aplicação de nitrogênio com a serragem cromada (SC + NPK + Calcário) favoreceu inicialmente a decomposição deste resíduo, possivelmente em função da degradação do carbono nativo do solo. Após o período de 38 dias, entretanto, a taxa de decomposição foi menor do que no tratamento sem nitrogênio (SC + PK + Calcário).

$\mathrm{A}$ adição de resíduo carbonífero não afetou a liberação de $\mathrm{C}-\mathrm{CO}_{2}$ ( $\mathrm{RC}+\mathrm{NPK}+$ Calcário), que foi semelhante à determinada no tratamento com adubação mineral, embora tenha sido observado um decréscimo do $\mathrm{pH}$ no final do experimento (Tabela 4). A adição de lodo de curtume com resíduo carbonífero, entretanto, estimulou a liberação de $\mathrm{C}-\mathrm{CO}_{2}$, que foi significativamente maior do que a determinada no tratamento com adição de somente a mesma quantidade de lodo (Figura 1).

A adição de $500 \mathrm{~kg} \mathrm{ha}^{-1}$ de cromo trivalente na forma mineral $\left(\mathrm{LC}+\mathrm{PK}+\mathrm{Cr}_{\min }\right)$ reduziu a decomposição do lodo de curtume em $29 \%$ no período de 88 dias (Figura 1). Entretanto, a mesma quantidade total de cromo foi adicionada no tratamento com a aplicação de duas vezes a dose recomendada de lodo (2 LC+PK), obtendo-se neste tratamento a maior liberação de $\mathrm{C}-\mathrm{CO}_{2}$ do experimento. Possivelmente, a maior concentração de cromo afetou a atividade microbiana no tratamento com adição de cromo mineral. Isto é devido à forma em que o cromo está disponível aos microorganismos do solo. Se o cromo é adicionado via mineral, ele esta bem mais biodisponível aos microorganismos quando comparado ao resíduo de lodo de curtume, onde os microorganismos necessitam degradar o resíduo, mineralizando o cromo, e liberando teores de cromo mais baixos com relação ao cromo mineral, comparado ao mesmo período de adição.

\subsection{Nitrogênio mineral}

A mineralização é o processo de transformação do nitrogênio orgânico para formas inorgânicas como o $\mathrm{NH}_{4}^{+}$e $\mathrm{NO}_{3}^{-}$, por organismos heterotróficos do solo. A taxa de mineralização varia em função da relação $\mathrm{C} / \mathrm{N}$, pH, temperatura, umidade e disponibili- dade de nutrientes. A imobilização é a transformação do nitrogênio inorgânico para formas orgânicas. A mineralização e a imobilização do nitrogênio ocorrem simultaneamente no solo.

A determinação do teor de nitrogênio mineral $\left(\mathrm{NH}_{4}^{+}\right.$e $\left.\mathrm{NO}_{3}^{-}\right)$foi realizada no final do experimento (Tabela 4). Os maiores teores de nitrogênio mineral foram observados nos tratamentos com adubação nitrogenada (NPK + Calcário, SC + NPK + Calcário e $\mathrm{RC}+\mathrm{NPK}+$ Calcário). Embora os resíduos de curtume tenham apresentadas relações $\mathrm{C} / \mathrm{N}$ de 6,3 e 2,7 para o lodo e a serragem cromada, respectivamente, as quantidades de nitrogênio mineral determinadas no final do experimento foram menores. Possivelmente devido ao processo de curtimento do couro que torna este material muito recalcitrante pela presença do cromo. Esse aspecto pode ser explicado pela baixa taxa de decomposição nesses tratamentos que provavelmente tenha liberado as menores quantidades de $\mathrm{N}$ mineral (Figura 1). Metais pesados e materiais recalcitrantes de difícil decomposição retardam a mineralização do material orgânico ou pela toxicidade ou pela baixa taxa de decomposição dos materiais (MOREIRA e SIQUEIRA, 2006).

Tabela 4. Teores de $\mathrm{NH}_{4}^{+}$e $\mathrm{NO}_{3}^{-}$e $\mathrm{pH}$ em água do solo após 88 dias de incubaçã̃o (média de três repetições)

\begin{tabular}{|c|c|c|c|}
\hline Tratamento & $\mathrm{NH}_{4}^{+}$ & $\mathrm{NO}_{3}^{-}$ & $\mathrm{pH}$ \\
\hline & \multicolumn{2}{|c|}{---- $\mathrm{mg} \mathrm{kg}^{-1}$} & \\
\hline 1 - Testemunha & $8,4 \mathrm{a}$ & $58,5 \mathrm{~h}$ & $4,5 \mathrm{~h}$ \\
\hline 2 - NPK + calcário & $0,8 \mathrm{c}$ & $271,0 \mathrm{a}$ & $6,3 \mathrm{e}$ \\
\hline $3-\mathrm{LC}+\mathrm{PK}$ & $1,5 \mathrm{c}$ & $157,4 \mathrm{e}$ & $7,1 \mathrm{c}$ \\
\hline $4-2 \times \mathrm{LC}+\mathrm{PK}$ & $2,1 \mathrm{bc}$ & $208,8 \mathrm{c}$ & $7,8 \mathrm{a}$ \\
\hline $5-\mathrm{RC}+\mathrm{NPK}+$ calcário & $6,2 \mathrm{ab}$ & $248,6 \mathrm{~b}$ & $4,8 \mathrm{~g}$ \\
\hline $6-\mathrm{RC}+\mathrm{LC}+\mathrm{PK}$ & $1,2 \mathrm{c}$ & $176,4 \mathrm{~d}$ & $7,5 \mathrm{~b}$ \\
\hline $7 \mathrm{a}-\mathrm{SC}+\mathrm{NPK}+$ calcário & $2,0 \mathrm{bc}$ & $267,4 \mathrm{a}$ & $6,0 \mathrm{f}$ \\
\hline 7b - SC + PK + calcário & $1,1 \mathrm{c}$ & $98,1 \mathrm{~g}$ & $6,7 \mathrm{~d}$ \\
\hline $8-\mathrm{Cr}+\mathrm{LC}+\mathrm{PK}$ & $3,9 \mathrm{abc}$ & $138,6 \mathrm{f}$ & $6,8 \mathrm{~d}$ \\
\hline
\end{tabular}

* Médias seguidas de mesma letra minúscula não diferem entre tratamentos pelo teste de Tukey $(\mathrm{p}<0,05)$. 
No tratamento com adição de lodo de curtume $(\mathrm{LC}+\mathrm{PK})$ foi determinada a quantidade de $318 \mathrm{~kg}$ de $\mathrm{N}$ mineral ha- ${ }^{-1}$ aos 88 dias, superior à adicionada pelo adubo mineral. Mesmo no tratamento com adição de serragem cromada (SC + PK+Calcário) a disponibilidade total de $\mathrm{N}$ (do solo e mineralizado) foi de $200 \mathrm{~kg}$ ha $^{-1}$ aos 88 dias. Possivelmente devido ao efeito priming em função da adição de corretivos e fertilizantes e revolvimento do solo para incorporar os resíduos.

A adição de cromo mineral ( $\left.\mathrm{LC}+\mathrm{PK}+\mathrm{Cr}_{\min }\right)$ reduziu em $15 \%$ a liberação de nitrogênio (Tabela 4 ), pelo efeito negativo sobre a atividade microbiana, conforme foi constatado pela volatilização de $\mathrm{C}-\mathrm{CO}_{2}$. As condições da incubação favoreceram a nitrificação tanto do nitrogênio adicionado pela uréia como do mineralizado dos resíduos. A fração média de 98,3\% do nitrogênio mineral do solo foi determinada na forma de $\mathrm{NO}_{3}$.

\section{4. $\mathrm{pH}$ do solo}

A aplicação de calcário (NPK + Calcário) aumentou o $\mathrm{pH}$ do solo de 4,5 para 6,3 (Tabela 4). A adição de lodo de curtume na quantidade recomendada (LC+PK) elevou o pH do solo para 6,1. A aplicação de duas vezes esta quantidade mostrou um aumento excessivo do $\mathrm{pH}$, o que deve ser evitado, podendo provocar deficiência de fósforo e de micronutrientes para as plantas. A elevação do $\mathrm{pH}$ do solo pela adição de resíduos orgânicos é frequentemente observada. Alguns autores (CASTILHOS et al., 2002; KRAY et al., 2008) atribuem este efeito à presença de compostos de $\mathrm{Ca}$ e de $\mathrm{Mg}$ no material e da presença de $\mathrm{NaOH}$ usada no processo de curtimento.

$\mathrm{O}$ pH do solo nos tratamentos com adição de resíduo carbonífero foi inferior ao determinado no tratamento com adubação mineral + calagem. Este decréscimo pode ser devido à oxidação da pirita contida no resíduo, à semelhança do que foi observado por Kray et al. (2008) em experimento de campo (Argissolo vermelho distrófico típico). A velocidade de oxidação da pirita durante a incubação foi maior do que a observada a campo por Kray et al. (2008), devido à menor granulometria do material e condições de aeração, temperatura e umidade mais favoráveis no experimento em laboratório.

\section{Conclusões}

O resíduo carbonífero apresenta grande potencial de acidificação do solo e baixa taxa de decomposição.
O lodo de curtume foi eficiente para aumentar o $\mathrm{pH}$ do solo e suprimento de nitrogênio para o solo. O lodo de curtume pode ser utilizado para a neutralização da acidez do solo gerada pelo descarte de resíduo carbonífero contendo alto teor de enxofre reduzido (pirita).

Devido à recalcitrância da serragem cromada, é necessário calagem e adubação com nitrogênio, fósforo e potássio em quantidades adequadas para o desenvolvimento das culturas, além disso, este resíduo apresentou potencial para descarte no solo.

\section{Agradecimentos}

A CAPES e ao CNPq pelo apoio financeiro e bolsas concedidas.

\section{Referências.}

ATLAS, R.M.; BARTHA, R. Microbial Ecology: Fundamentals and Applications. $4^{\text {th }}$ ed. Menlo Park: Benjamin/Cummings Science Publishing, 1997. 694p.

BIANCHIN, L. Atributos químicos e especiação de cromo em solo com aplicação de resíduos de curtume e carbonífero em experimento de campo. 2011. 114f. Tese (Doutorado) - Programa de Pós Graduação em Ciência do Solo, Faculdade de Agronomia, Universidade Federal do Rio Grande do Sul, Porto Alegre, 2011.

CASTILHOS, D.D.; TEDESCO, M.J.; VIDOR, C. Rendimentos de culturas e Alterações químicas do solo tratado com resíduos de curtume e cromo hexavalente. Revista Brasileira Ciência do Solo, v.26, p.1083-1092, 2002.

CAVALLET, L.E.; SELBACH, P.A.; GIANELLO, C. Concentração de cromo no sistema solo-plantapercolado em função da aplicação de resíduos de curtume em um argissolo de estância velha (RS). Scientia Agraria, v.8, p.87-93, 2007.

COLLA, T.S.; ANDREAZZA, R.; BÜCKER, F.; SOUZA, M.M.; TRAMONTINI, L.; PRADO, G.R.; FRAZZON, A. P. G.; CAMARGO, F.A.O. ; BENTO, F.M. Bioremediation assessment of dieselbiodiesel-contaminated soil using an alternative bioaugmentation strategy. Environmental Science and Pollution Research International, New York, 2013. doi: 0.1007/s11356-013-2139-2. 
EMBRAPA. Sistema brasileiro de classificação de solos. Brasília: Embrapa-SPI, 2006. 412 p.

FERREIRA, A.S.; CAMARGO, F.A.O.; TEDESCO, M.J.; BISSANI, C.A. Alterações de atributos químicos e biológicos de solo e rendimento de milho e soja pela utilização de resíduos de curtume e carbonífero. Revista Brasileira Ciência do Solo, v.27, p.1083-1092, 2003.

FIGLIOLIA, A.; BENEDETTI, A.; DELL'ABATE, M.T.; IZZA, C.; INDIATI, R. Potential chromium bio-availability by Lactuca sativa grown on two soils amended with tannery leather residues.

Fresenius Environmental Bulletin, v.1, p.406-410, 1992.

FISCH, J. Lodo cromado: projeto demonstra viabilidade de utilização em solo agrícola. Setor Couro, v.8, p.6-15, 1992.

FISCH, J. Utilização de lodo de curtimento ao cromo em solo agrícola. Revista Couro, v.20, p.6687, 1994.

HERNANDEZ, T.; GARCIA, C.; LAX, A. Transformation of carbon and nitrogen in a calciorthid soil amended with a range of organic residues. Plant Soil, v.105, p.205-211, 1988.

KRAY, C.H.; TEDESCO, M.J.; BISSANI, C.A.; GIANELLO, C.; SILVA, K.J. Tannery and coal mining residues disposal on soil. Revista Brasileira Ciência do Solo, v.32, p.2877-2882, 2008.

KRAY, C.H.; TEDESCO, M.J.; BISSANI, C.A.; BORTOLON, L.; ANDREAZZA, R.; GIANELLO, G. Aplicação de composto de lixo urbano e lodo de esgoto em dois solos diferentes. Pesquisa Agropecuária Gaúcha, v.17, p.119-125, 2011.

KROB, A.D.; MORAES, S.P.; SELBACH, P.A.; BENTO, F.M.; ANDREAZZA, R.; CAMARGO, F.A.O. Alterações nas propriedades físicas e microbiológicas do solo sob adição contínua de composto de lixo urbano. Pesquisa Agropecuária Gaúcha, v.17, p.137-144, 2011.

LAUSCHNER, M.H.; TEDESCO, M.J.; GIANELLO, G.; BORTOLON, L.; KRAY, C.H.; BARBOSA, D.B.P.; ANDREAZZA, R. Utilização de resíduos de agroindústria fumageira como corretivo de acidez em diferentes solos. Pesquisa Agropecuária Gaúcha, v.18, 75-80, 2012.
MEYER, D.D.; SANTESTEVAN, N.A.; BÜCKER, F.; SALAMONI, S.P.; ANDREAZZA, R.; CAMARGO, F.A.O.; BENTO, F.M. Capability of a selected bacterial consortium for degrading diesel/ biodiesel blends (B20): enzyme and biosurfactant production. Journal of Environmental Science and Health. Part A, Toxic Hazardous Substances and Environmental Engineering, Amsterdam, v.47, p.1776-1784, 2012.

MOREIRA, F.M.S.; SIQUEIRA, J.O. Microbiologia e Bioquímica do Solo. 2 ed. Lavras: Editora UFLA, 2006. 729p.

SBCS/NRS (Sociedade Brasileira de Ciência do Solo/Núcleo Regional Sul). Recomendações de adubação e de calagem para os estados do Rio Grande do Sul e de Santa Catarina. 3. ed. Passo Fundo: Embrapa-CNPT, 1995. 224p.

SEGATTO, M.P. Efeitos da aplicação de resíduos industriais no solo e nas plantas. 2001. 151f. Dissertação (Mestrado) - Programa de PósGraduação em Ciência do Solo, Faculdade de Agronomia, Universidade Federal do Rio Grande do Sul, Porto Alegre, 2001.

SEGATTO, M.P.; ANDREAZZA,R.; BORTOLON, L.; SANTOS, V.P.; GIANELLO, C.; CAMARGO, F.A.O. Decomposição de resíduos industriais no solo. Ciência e Natura, v.34, p.49-62, 2012.

SELBACH, P.A; TEDESCO, M.J.; GIANELLO, C. Descarte e biodegradação de lodos de curtume no solo. Revista Couro, v.17, p.51-62, 1991.

STOMBERG, A.L.; HEMPHILL, D.D. VOLK, V.V. Yield and elemental concentration of sweet corn grown on tannery waste-amended soil. Journal of Environmental Quality, v.13, p.162-166, 1984.

STOTZKY, G. Microbial respiration. In: BLACK, C.A., (Ed) Methods of soil analysis. Madison: American Society of Agronomy, 1965. p. 15511572. Pt. 2: Chemical and microbiological proprieties (Agronomy Series, 9).

TEDESCO, M.J.; GIANELLO, C.; BISSANI, C.A.; BOHNEN, H.; VOLKWEISS, S.J. Análises de solos, plantas e outros materiais. 2.ed Porto Alegre: Departamento de Solos da Faculdade de Agronomia, UFRGS,. 1995. 174p. (Boletim Técnico, $5)$. 
TEIXEIRA, J.A.O.S. Descarte de resíduo de curtume no solo. 1981. 81f. Dissertação (Mestrado) - Programa de Pós Graduação em Ciência do Solo, Faculdade de Agronomia, Universidade Federal do Rio Grande do Sul, Porto Alegre, 1981.

ZIBILSKE, L.M. Dynamics of nitrogen and carbon in soil during peppermill sludge decomposition. Soil Science, v.143, n.1, p. 26-33, 1987. 